\title{
One Orthogonal Phase Microscopy Technique for Orthogonal Phase Imaging of a Cell
}

\author{
Jing-ye LIU1 ${ }^{1}$, Yuan-yuan XU1 ${ }^{1}$, Qiong ZHU ${ }^{1}$, Meng-yuan SHANG ${ }^{2}$, \\ Jing-rong $\mathrm{LIAO}^{3}$ and Ya-wei WANG ${ }^{1, a, *}$ \\ ${ }^{1}$ Faculty of Science, Jiangsu University, Zhenjiang, China \\ ${ }^{2}$ Affiliated Hospital, Jiangsu University, Zhenjiang, China \\ ${ }^{3}$ School of Automotive and Traffic Engineering in Jiangsu University, China \\ ajszjwyw@sina.cn \\ ${ }^{*}$ Corresponding author
}

Keywords: Biological cells, Quantitative phase imaging (QPI), Orthogonal optical path, Off-axis interference, 3D rebuilding, Phase retrieval.

\begin{abstract}
For human beings, the morphological features of cells are closely related to human health thus research into the technique of cell imaging is very important. The optical phase imaging technique is one of the most useful methods because the cell is a phase object. More than one phase images should be needed when rebuilding the 3D sub-structure of a cell. In this paper, in order to obtain the complete 3D information of a cell under the maximum entropy tomography method, two orthogonal phase images is acquired simultaneously, so one orthogonal interference imaging system based on dual orthogonal optical path is constructed by using a lateral displacement beam splitter is proposed. It can obtain two interference images at the same time on the orthogonal direction, so that the biological cellular 3D morphology and its sub-structure can be obtained by using some phase recovery method. Compared with the traditional off-axis interferometry, the full information of the cell is omitted in this method. The simulation and experiment results show the validity and stability of the proposed method. This technique could be widely applied to phase microscopy, especially for transparent samples, such as phase imaging for biological cells and phase measurement, etc.
\end{abstract}

\section{Introduction}

With the development of the theoretical research in human life science, the requirement of the method and the technique, which can be used to detect the cell, becomes more and more significant. In connection with this, optics is one of the oldest and most promising subjects, which provision of a new research perspective and means for the scientific research of life science. Especially the development of all kinds of microscopy that provides people with a new perspective to study the phenomena of life activities. At the same time, the new rules and findings has been explored by life science researchers in the microscopic field of the biological molecules, cells, tissues and organs. It becomes necessary for researchers to pay more attention to the more powerful technique which they need. Thus optical microscopy should consider the requirements in developing new detection methods, which have the advantages of real-time, high sensitivity, high resolution and no damage.

Because cells have the characteristic of transparency, and the traditional optical microscope cannot observe their structure information clearly, phase microscopy technique, which makes full use of intrinsic refractive index of the cell to increase the 
contrast, has attracted a lot of attention. Especially, over the past decades, a lot of advanced phase microscopy techniques [1-5] have been proposed to realize the quantitative phase imaging with high accuracy. With this techinque, the shape and dynamics of cells are studied on the basis of the phase information. Unfortunately, for many quantitative phase microscopy techniques, only two dimensional phase information can be obtained, which can not reflect sub-structure information of the real cell.

Tomography phase microscopy (TPM) [6-10] is an emerging technique that provides quantitative refractive index distributions and 3D structure information of biological cells. A common idea of TPM is to record a set of quantitative phase images at various illumination angles, and then to reconstruct 3D structure with these images according to the corresponding algorithm. For example, in 2007, Choi et al. [6] proposed a high-resolution TPM based on a phase-shifting laser interferometric microscope. In this technique, a galvanometer scanning mirror is required to vary the illumination angle of the light source for acquiring phase images in multiple directions, and the filtered back-projection method is applied to reconstruct a $3 \mathrm{D}$ refractive index tomogram. This technique can obtain the satisfying results for the thin specimens, such as live cells and tissues. In Ref. [9], a TPM is based on the digital holographic phase microscopy. The phase projection data in multi-directions can be obtained by rotating the specimen under a fixed illumination beam, and then a filtered back projection procedure is applied to deal with these data for achieving the complete projection reconstruction of the refractive index. In Ref. [10], the optical tweezers is introduced to a phase-shifting Mach-Zehnder interferometer for achieving the TPM. In this interferometer, two light sources are used, among which the light wave induced by the green laser can control the cell rotation. This TPM realizes the measurement of cell at the total solid angle range of $360^{\circ}$, thus it can overcome a common problem existing in some TPM methods, that is the reconstructed error caused by the insufficient projection data.

In summary, a series of phase images under different illumination angles are required in these tomography methods mentioned above. To reduce the measuring data, in this paper, we propose an orthogonal phase microscopy technique, with imaging from two orthogonal directions. Then using the maximum entropy tomography method, we reconstruct the refractive index and the $3 \mathrm{D}$ sub-structure from the phase distribution of the two vertical directions. [11, 12]

\section{Orthogonal Phase Microscopy Imaging System}

The design of one orthogonal phase microscopy technique is shown in Figure 1, in which, beam from the laser pass through the beam splitter (BS), is splitted into two beams, horizontal and vertical, and then the horizontal beam pass through the lateral displacement beam spiliter one (LDBS1) into two parallel beams. One of them carries the sample information, when transmitted through the sample, into the LDBS2 with another beam, which comes from the LDBS1. Thus the interference pattern is obtained by the CMOS1 on its screen. Under a certain distance from the BS the vertical beam, which comes from the BS, is refracted through the mirror 1(M1) to the horizontal direction. Then it passes through the LDBS3 into two parallel beams, one of them transmits through the sample, as the object beam. Another one directly incidents onto the LDBS4, as the reference beam. These two beams are combined into one part by transmission and reflecting of the LDBS4. Finally, the interferogram is recorded by the CMOS2. It is worth noting that the two sensors obtain the 
interferograms simultaneously.

Using the Hilbert transform, the phase distribution is obtained, the phase information of the sample is demodulated, and finally a three-dimensional morphology of the sample can be reconstructed based on the unwrapped phase.

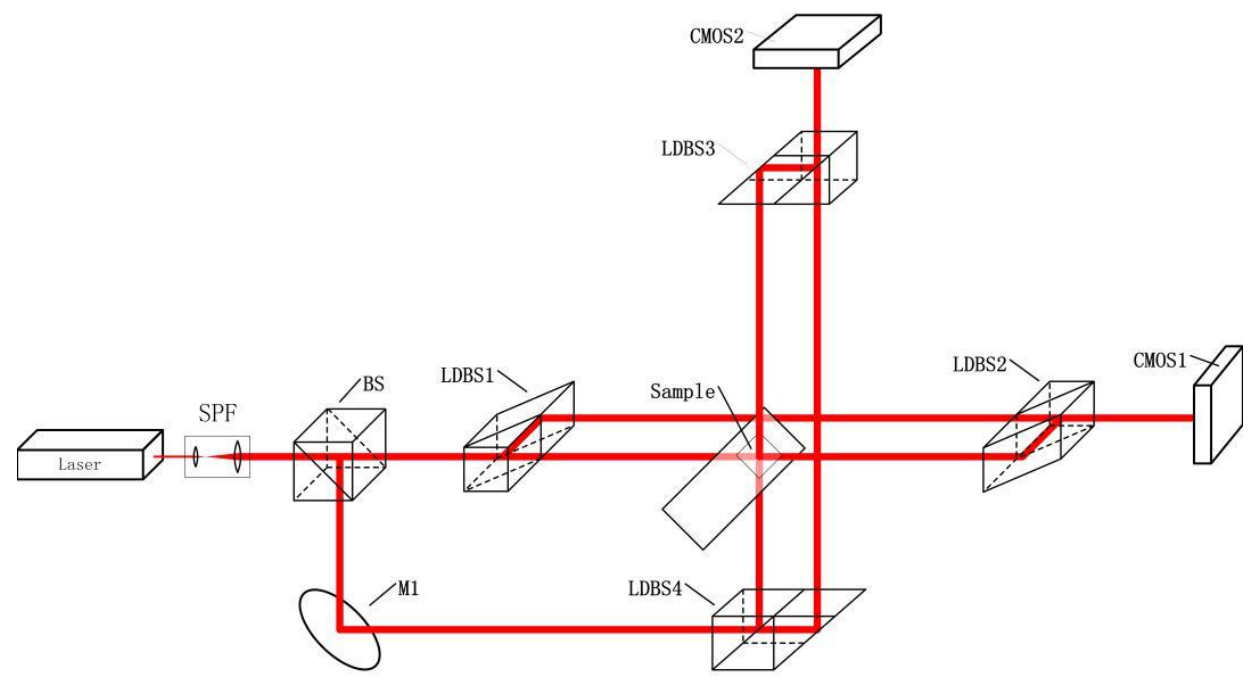

Figure 1. The experimental setup

\section{Phase Retrieval Methods}

In the off-axis interferometry, since the object and reference waves are not parallel, the linear phase factor is introduced, and the phase can be retrieved only by one single interferogram. The interference intensity can be given by

$$
I(x, y)=I_{0}(x, y)+\gamma(x, y) \cos [\varphi(x, y)+k x]
$$

Here, $\quad I_{0}(x, y)=|O(x, y)|^{2}+|R(x, y)|^{2} \quad$ is the background intensity, $\gamma(x, y)=2|O(x, y) \| R(x, y)| \quad$ is the amplitude modulation factor, and $\varphi(x, y)=\varphi_{O}(x, y)-\varphi_{R}(x, y)$ is the relative phase difference between the object and reference waves. $k$ is the spatial frequency of the fringes. In this case, the background intensity can be eliminated by a high pass filter or an average algorithm. Consequently, we can obtain the interference term, which is given by

$$
I^{(0)}=\gamma \cos [\varphi(x, y)+k x]
$$

Then, the interference term is processed by applying the fast derivative algorithm. Considering the real case, we have the following approximation:

$$
\frac{\partial I_{0}}{\partial x} \approx 0, \frac{\partial \gamma}{\partial x} \approx 0, \frac{\partial^{2} \varphi}{\partial x^{2}} \approx 0
$$

This approximation of the phase is easily satisfied, even for some complex structured phase objects. Thus, the 1st and 2nd order derivatives of Eq. 2 can be approximated as

$$
I^{(1)}=-\gamma \sin [\varphi(x, y)+k x]\left(\frac{\partial \varphi}{\partial x}+k\right)
$$




$$
I^{(2)}=-\gamma \cos [\varphi(x, y)+k x]\left(\frac{\partial \varphi}{\partial x}+k\right)^{2}
$$

Then, according to Eqs. 2, 4 and 5, the phase of a sample can be expressed as

$$
\varphi(x, y)= \begin{cases}\tan ^{-1}\left\{\left[-\frac{\left[I^{(1)}\right]^{2}}{I^{(2)} \cdot I^{(0)}}\right]^{1 / 2}\right\}-k x & \left(I^{(1)} \cdot I^{(2)} \geq 0\right) \\ \tan ^{-1}\left\{-\left[-\frac{\left[I^{(1)}\right]^{2}}{I^{(2)} \cdot I^{(0)}}\right]^{1 / 2}\right\}-k x & \left(I^{(1)} \cdot I^{(2)} \geq 0\right)\end{cases}
$$

\section{Simulation and Experimental Validation}

In order to demonstrate the ability of the orthogonal phase microscopy technique, we carry out the simulation and experiment of a tiny ball.

In our simulation, the radius and the refractive index of the ball are set as $2 \mu \mathrm{m}$ and 1.59. Other parameters are as follows: the refractive index of the environment liquid is 1.57, the amplitudes of the object and reference waves are unit, the wavelength of the light source is $632.8 \mathrm{~nm}$, the spatial frequency $\mathrm{k}$ is $1.0683 \mathrm{rad} / \mathrm{pixel}$, and the size of the interference pattern recorded by a CCD is $512 \times 512$ pixels. According to these associated parameters and the phase retrieval theory in off-axis interferometry mentioned in Section 3, we can perform the phase imaging quantitatively. Fig. 2(a) is the interferogram. Fig. 2(b) is the interference term after high-pass filtering. Figs. 2(c) and 2(d) are the 1st and 2nd order derivatives of Fig. 2(a). According to Eq. 6, we can obtain the phase distribution of the ball, as shown in Fig. 2(e). Since the ball is homogeneous, there is a linear relationship between the axial thickness and phase distribution. To be specific, assuming that the light propagates along $\mathrm{z}$-axis, this relationship can be expressed as

$$
\varphi(x, y)=\frac{2 \pi}{\lambda} \cdot \Delta n \cdot H=\frac{2 \pi}{\lambda} \cdot \Delta n \cdot\left[2 \sqrt{R^{2}-x^{2}-y^{2}}\right]
$$

In which, $H$ is the thickness along with the optical axis, and $\Delta n$ is the refractive index difference between the ball and the environment liquid. So the thickness information can be calculated from the measured phase directly, and the result is presented in Fig. 2(f). Via the calculation, the maximum thickness of the ball is $4.0072 \mu \mathrm{m}$, and the corresponding error is $0.0072 \mu \mathrm{m}$, which is very small compared with the theoretical value. It shows that this phase retrieval method is feasible and highly accurate. 

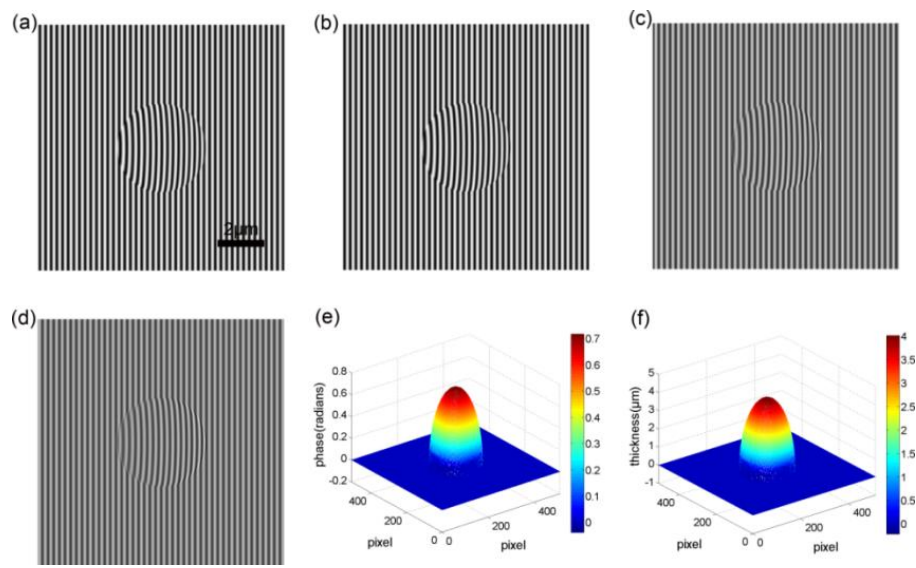

Figure 2. Simulation experiment. (a) interfeogram, (b) interference item, (c) the first order derivative of (a), (d) the second orderderivativeof (a), (e) the reconstruction phase, (f) the reconstruction thickness

In the experiment, we carry out the phase imaging of a $\mathrm{SiO}_{2}$ ball with the diameter of $1.5 \mathrm{~mm}$. The experimental setup is shown in Fig. 1. The parameters of main components are as follows: A He-Na laser with the wavelength of $632.8 \mathrm{~nm}$ is selected as the light source, the beam diameter after extending is $10 \mathrm{~mm}$, the resolution of the CMOS (Infinity 1) is $1024 \times 1024$ pixels, a lateral displacement beam spiliter ( $450 \mathrm{~nm} \sim 650 \mathrm{~nm}$ ) is used to split or combine the object beam and reference beam, a beam spiliter $(450 \mathrm{~nm} \sim 650 \mathrm{~nm})$ is used to split the beam into two different directions, and the parameters of the reflecting mirror is $R_{\text {avg }}>99.7 \%$ and $520 \mathrm{~nm} \sim 647 \mathrm{~nm}$.

The experimental result is shown in Fig.3 based on the principle of Fig.1. Fig.3(a) shows the micrograph of a $\mathrm{SiO}_{2}$ ball, Figs. 3(b) and 3(c) show the interferograms on different directions of the horizontal and vertical, Fig. 3(d) shows the phase distribution which was calculated from the horizontal direction interferogram. (Because it is the same as Fig. 3(d) the phase of the vertical direction is omitted.) Comparing the results from experiment and simulation, it is clear that there is basic agreement between the two phase distributions.
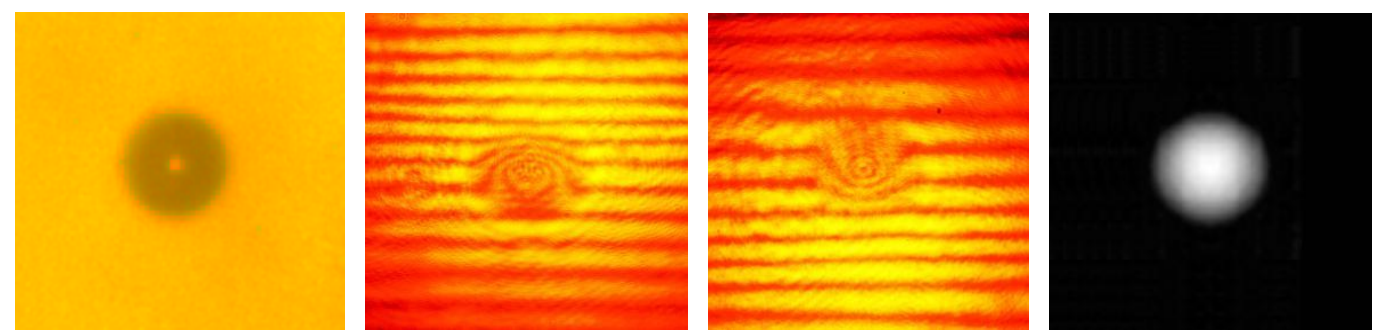

Figure 3. Experimental results of a $\mathrm{SiO}_{2}$ ball. (a) the micrograph, (b) the interferogram on horizontal direction, (c) the interferogram on vertical direction, (d) the reconstructed phase

\section{Conclusion}

In summary, this paper proposes a technique of orthogonal phase microscopy. This technique has the advantage of simplicity and stability, because the Mach-Zehnder interferometer can be easily realized. Compared with the traditional Mach-Zehnder interferometer, two orthogonal phase images of the sample is easily obtained by the two orthogonal interferogram. The phase and thickness distribution can be calculated with the maximum entropy tomography method. The simulation and experiment results both show that the images obtained by this method have good stability and resolution ratio. It may have a good application in QPI field. 


\section{Acknowledgement}

This work was supported by National Natural Science Foundation of China (Nos. 11604127, 11374130 and 11474134).

\section{Reference}

[1] G. Popescu, L.P. Deflores, J.C. Vaughan, K. Badizadegan, H. Iwai, R.R. Dasari, M.S. Feld, Fourier phase microscopy for investigation of biological structures and dynamics, Opt. Lett. 29 (2004) 2503-2505.

[2] G. Popescu, T. Ikeda, R.R. Dasari, M.F. Feld, Diffraction phase microscopy for quantifying cell structure and dynamics, Opt. Lett. 31 (2006) 775-778.

[3] N.T. Shaked, Y. Zhu, M.T. Rinehart, A. Wax, Two-step-only phase-shifting interferometry with optimized detector bandwidth for microscopy of live cells,Opt. Express 17 (2009) 15585-15591

[4] N.T. Shaked, M.T. Rinehart, A. Wax, Dual-interference-channel quantitative-phase microscopy of live cell dynamics, Opt. Lett. 3 (2009) 767-769.

[5] H.V. Pham, B. Bhaduri, K. Tangella, C. Best-Popescu, G. Popescu, Real time blood testing using quantitative phase imaging, Plos. One 8 (2013) e55676.

[6] W. Choi, C. Fang-Yen, K. Badizadegan, S. Oh, N. Lue, R.R. Dasari, M.S. Feld,Tomographic phase microscopy, Nat. Methods 4 (2007) 717-719.

[7] P. Xiu, X. Zhou, C.F. Kuang, Y.K. Xu, X. Liu, Controllable tomography phase microscopy, Opt. Lasers Eng. 66 (2015) 301-306.

[8] A. Kus, M. Dudek, B. Kemper, M. Kujawinska, A. Vollmer, Tomographic phase microscopy of living three-dimensional cell cultures, J. Biomed. Opt. 19 (2014) 046009.

[9] F. Charrière, A. Marian, F. Montfort, J. Kuehn, T. Colomb, Cell refractive index tomography by digital holographic microscopy, Opt. Lett. 31 (2006) 178-180.

[10] T. Yasokawa, I. Ishimaru, M. Konodo, A method for measuring the three-dimensional refractive-index distribution of single cells using proximal two-beam optical tweezers and a phase-shifting Mach-Zehnder interferometer,Opt. Rev. 14 (2007) 161-164.

[11] Y.W. Wang, S.H. Liu, Y. Ji, Y.Y. Xu, W.F. Jin, H. Wu, S.L. Ding, An entropy method for 3D subsurface imaging of a nucleated cell based on orthogonal phase images, Opt. Commun. 326 (2014) 170-174

[12] Y.W. Wang, L. Zhang, Y. Ji, Y.Y. Xu, M. Bu, Y.Z. Chen, 3D morphological reconstruction of red blood cell based on two phase images, Opt. Appl. 45 (2015) $173-182$ 